\title{
Ciudades medias de Brasil: una tipología socioambiental
}

\section{Brazilian medium cities: a socio-environmental typology}

\begin{abstract}
Fabio Angeoletto - Doutor em Ecologia pela Universidade Autônoma de Madrid. Mestre em Arquitetura e Urbanismo pela Universidade Federal da Bahia. Professor do Programa de Pós-Graduação em Geografia da Universidade Federal do Mato Grosso (UFMT). Pesquisador do Grupo de Estudios en Ecología Humana, Urbana y del Paisage de la Universidad Autónoma de Madrid. fabio_angeoletto@yahoo.es
\end{abstract}

Camila Essy - Graduada em Farmácia e Bioquímica pela Universidade Federal de Santa Maria. Pesquisadora do Grupo de Estudios en Ecología Humana, Urbana y del Paisage de la Universidad Autónoma de Madrid. camilaessy@gmail.com

Juan Pedro Ruiz Sanz - Doutor em Ecología pela Universidad Autónoma de Madrid. Professor titular do curso de Doutorado en Ecología de la Universidade Autónoma de Madrid. Pesquisador del Grupo de Estudios en Ecología Humana, Urbana y del Paisage de la Universidade Autónoma de Madrid.juan.ruiz@uam.es

\section{Resumo}

La urbanización global de los territorios, que es el fenómeno ecológico humano más importante en curso, y cuyos impactos ambientales poseen niveles local, regional y mundial, presenta la característica notable de un crecimiento pronunciado de las ciudades pequeñas e intermedias, en las cuales la capacidad de planificación y gestión es usualmente ínfima, en comparación a las ciudades grandes. Es lo que se verifica en Brasil, donde las ciudades de tamaño medio, es decir, las que tienen poblaciones entre 100.000 y 500.000 vecinos están creciendo más rápidamente que las grandes ciudades, desde mediados de la década de 1990. El artículo tiene como objetivo trazar una tipología de las ciudades medias brasileñas por medio de análisis estadísticos multivariados. La tipología resultante señala dos tipos de ciudades, con demandas sociales, ambientales y de gestión diferentes. Algunas de estas ciudades se encuentran en hotspots de biodiversidad. En paralelo, hemos recopilado estadísticas sobre la gestión ambiental en marcha en estas ciudades. Nuestros resultados demuestran una baja efectividad de las acciones de gestión ambiental en estos municipios.

\section{Palavras-chave}

Ecología urbana. Gestión ambiental. Ciudades medias. Urbanización.

\begin{abstract}
The global urbanization of the territories, which is the most important ongoing human ecological phenomenon, and whose environmental impacts have local, regional and global levels, has the remarkable property of a sharp growth of small and intermediate cities, in which capacity of planning and management is usually negligible, when they are compared to large cities. This is what takes place in Brazil, where mediumsized cities, those with populations between 100,000 and 500,000 residents are growing faster than large cities from mid-1990. The article aims to chart a typology of medium Brazilian cities, through multivariate statistical analysis. The resulting typology identifies two types of cities, with different social, environmental and management demands. Some of these cities are located in biodiversity hotspots. In parallel, we have compiled statistics on environmental management in place in these cities. Our results demonstrate low effectiveness of environmental management actions in these municipalities.
\end{abstract}

\section{Keywords}

Urban ecology. Environmental management. Medium cities. Urbanization. 


\section{INTRODUCCIÓN}

La urbanización es el más importante proceso socioambiental de la actualidad. William Rees (1997) define la masiva migración de personas para las ciudades como el más significativo evento ecológico humano del siglo XX. También es consensual el enorme conjunto de impactos causados por ese proceso, en escala local, regional y global (TERRADAS, 2001; ANGEOLETTO, 2012). En 1900, solo un 10\% de la población global era urbana (GRIMM et al, 2008). En 1950 la población urbana global subió a un 18\%, y en 2003 alcanzó el 42\% (WORLDWATCH INSTITUTE, 2006). En 2050 habrá entre ocho a diez mil millones de seres humanos, dos tercios de los cuales vivirán en ciudades (COHEN, 2003). El 95\% del crecimiento de la población urbana ocurre en los países en desarrollo, cuyas ciudades reciben 5 millones de nuevos vecinos mensualmente (UN-HABITAT, 2008). En Brasil, el proceso de urbanización resultó en un desplazamiento de millones de personas desde las zonas rurales hacia las ciudades. En 1890 la población urbana de Brasil era cerca de 10\%. En pocas décadas, ese cuadro se invierte: hoy, 83\% de los brasileños viven en ciudades. En un primer ciclo, los emigrantes se han desplazado hacia las ciudades grandes.

Desde mediados de los años 90, se inicia un segundo ciclo de urbanización en Brasil, con un aumento considerable del número de ciudades medias (aquellas con poblaciones entre 100.000 y 500.000 habitantes), de personas viviendo en ellas y del área ocupada por ellas. Se puede observar nítidamente una disminución del área ocupada por las metrópolis, mientras que el total de las ciudades medias evoluciona, desde 1970 hasta 2000, de un 11,77\% para un 27,23\% del área total ocupada por las ciudades brasileñas. El número de ciudades medias también crece considerablemente en ese periodo, pasando de 40, en 1970, a 194, en 2000 (CARVALHO, 2003). Entre 2000 y 2010, esa expansión se mantuvo: mientras que ciudades pequeñas y grandes crecieron a tasas anuales semejantes $(1,06 \%$ y 1,07\%, respectivamente), las ciudades medias crecieron 1,51\% al año (IPEA, 2011). Actualmente hay 283 ciudades medias en Brasil, que en conjunto concentran aproximadamente el 25\% de la población brasileña (ANGEOLETTO, 2012). Con relación al panorama de la urbanización brasileña, hemos trabajado para establecer una tipología de las tendencias socioambientales de las ciudades medias de Brasil, y un cuadro de la efectividad de las acciones de gestión ambiental allí practicadas, con el objetivo de auxiliar al establecimiento de políticas ambientales urbanas más exitosas en la protección de la biodiversidad y en el incremento de la calidad de vida de la gente. 


\section{MATERIALES Y MÉTODOS}

Para explorar los estándares existentes en un conjunto de objetos o entidades de los cuales se hayan tomado mediciones de una manera analítica y cuantitativa, han sido creados diversos procedimientos estadísticos exploratorios, conocidos como "análisis multivariados" (PRADO et al., 2001). Los análisis multivariados tienen como meta la reducción de un gran número de variables a pocas dimensiones, con un mínimo de pérdida de información, permitiendo la detección de los principales estándares de similitud, de asociación y de correlación entre dichas variables. El método de la ordenación multivariada es cualquier proceso que proporcione un pequeño número de variables que describan las relaciones entre objetos a partir de mediciones tomadas de esos objetos (ciudades, por ejemplo) (PRADO et al., 2001). El objetivo de la ordenación es reducir, sin pérdida de información, un gran número de mediciones en pocas variables sintéticas, facilitando la identificación de tendencias. En la mayoría de las técnicas de ordenación, las variables sintéticas, llamadas ejes o componentes, son combinaciones lineares de mediciones del tipo: $Y=a 1 \times 1+a 2 \times 2+a 3 \times 3$ $+\ldots a n x n$, donde xi son las mediciones y ai sus coeficientes lineares que indican su importancia para el eje. La participación de cada medición en un eje puede también ser medida por su peso (loading), que indica su correlación con el eje. La coordenada de cada objeto en un eje es dado, como indica la fórmula, por Y, lo que permite usar los ejes en un plano cartesiano para representar relaciones multivariadas en pocas dimensiones (PRADO et al., 2001).

El análisis de componentes principales (de aquí en adelante identificado por el acrónimo ACP) es, de los métodos de ordenación, el indicado para conjuntos de medidas correlacionados linealmente. Cuanto mayor es la correlación entre las mediciones originales, mayor será la parcela de variación explicada por los primeros ejes. Los coeficientes de las variables, o sus pesos, de mayor valor, ya sean positivos o negativos, son utilizados para interpretar los ejes.

También hemos procedido a un análisis estadístico univariado respecto de los datos de gestión ambiental del universo de las ciudades investigadas, comparándolos a los datos de gestión de las ciudades seleccionadas a través del análisis de componentes principales. Las informaciones para los análisis estadísticos han sido obtenidas en dos bancos de datos: "Pesquisa de Informações Básicas Municipais - Perfil dos municipios brasileiros, meio ambiente 2002", disponibles en la página web del IBGE (Instituto Brasileiro de Geografia e Estatistica) (http:/ /www. ibge.gov.br/home/estatistica/economia/perfilmunic/meio_ambiente_2002/ meio_ambiente2002.pdf), y a través de la página web IBGE Cidades (http:/ / www. cidades.ibge.gov.br/xtras/home.php), ambas accedidas en septiembre de 2014 . 
El banco de datos Perfil dos municipios brasileiros: meio ambiente 2002 (IBGE, 2005) contiene informaciones sobre diferentes categorías de gestión ambiental (por ejemplo, gestión de recursos hídricos y gestión de recursos forestales) y su ocurrencia en las ciudades brasileñas. También están cuantificados datos institucionales como el número de funcionarios de las secretarías municipales de medio ambiente y la ocurrencia de impactos ambientales diversos, como episodios de contaminación atmosférica, deforestación y otros. El Perfil es el único documento producido por el IBGE con esta amplitud de datos relativos a cuestiones ambientales urbanas. En la página web IBGE Cidades hemos recolectado datos socioeconómicos de las ciudades medias investigadas.

Para el análisis de componentes principales, hemos compilado en una planilla del software Excel 127 variables, de las 283 ciudades medias. Las variables han sido divididas del siguiente modo: variables ambientales (número de impactos ambientales, número de acciones de gestión ambiental), variables socioeconómicas (población, renta, escolaridad, coches privados, autobuses, motocicletas por grupos de 100.000 habitantes, entre otras).

\section{RESULTADOS Y DISCUSIÓN}

El análisis de componentes principales revela dos grupos bien diferenciados: el de las ciudades pobres y ricas ${ }^{1}$ (cuadros 1 y 2). Esta información, recogida en los dos primeros ejes, explica el 77,95\% de varianza acumulada (es decir, un 57,19\% de varianza en el eje 01; y un 20,76\% en el eje 02). El primer grupo lo forman aquellas ciudades con valores negativos para el factor 1 y positivos para el factor 2, cuyas características más notables son un gran porcentaje de analfabetos, poblaciones jóvenes, trabajadores con bajos sueldos y pocas acciones municipales de gestión ambiental. Este grupo de ciudades pobres está íntegramente concentrado en las regiones Norte / Nordeste de Brasil, con la excepción de Águas Lindas de Goiás, localizada en la región Centro-Oeste, cerca de Brasilia, la capital federal brasileña (Cuadro 1).

Otra excepción notable es la presencia de la ciudad de Itapevi entre las más pobres, ya que ella está ubicada en el Estado de São Paulo, el más rico de Brasil (las ciudades ricas están ubicadas en las regiones Sur y Sudeste). Se

\footnotetext{
Nos referimos a las ciudades seleccionadas a través del ACP como pobres y ricas, siempre señalándolas en cursiva, para resaltar las diferencias económicas entre ellas, aunque, evidentemente, existan distintas clases sociales en ambos grupos de ciudades.
} 
tratan de ciudades que, a pesar de su proximidad con regiones metropolitanas económicamente importantes, concentran pobreza e impactos ambientales correlacionados. Esas ciudades reproducen, así, el fenómeno común en regiones metropolitanas brasileñas: una relativa prosperidad en las ciudades-polo de las regiones, se traduce en la escasez de recursos y varias otras inequidades (incluso de matiz ambiental) en las ciudades del entorno.

Cuadro 1 - Ciudades pobres del ACP.

\begin{tabular}{|c|c|c|}
\hline Ciudades pobres & Bioma & Región \\
\hline Abaetetuba & Amazonia & Norte \\
\hline Açailândia & Amazonia & Nordeste \\
\hline Cametá & Amazonia & Norte \\
\hline Castanhal & Amazonia & Norte \\
\hline Macapá & Amazonia & Norte \\
\hline Marabá & Amazonia & Norte \\
\hline Parintins & Amazonia & Norte \\
\hline Santarém & Amazonia & Norte \\
\hline São José de Ribamar & Amazonia & Nordeste \\
\hline Águas Lindas de Goiás & Cerrado & Centro-Oeste \\
\hline Caxias & Cerrado & Nordeste \\
\hline Codó & Cerrado & Nordeste \\
\hline Timon & Cerrado/Caatinga & Nordeste \\
\hline Caucaia & Caatinga & Nordeste \\
\hline Itapipoca & Caatinga & Nordeste \\
\hline Arapiraca & Caatinga/ Mata Atlántica & Nordeste \\
\hline Itapevi & Mata Atlántica & Sudeste \\
\hline Nossa Senhora do Socorro & Mata Atlántica & Nordeste \\
\hline Santa Rita & Mata Atlántica & Nordeste \\
\hline Simões Filho & Mata Atlántica & Nordeste \\
\hline
\end{tabular}

Fuente: elaboración propia.

Por otro lado, las ciudades con valores positivos para factor 1 son las ciudades ricas, con poblaciones adultas y escolarizadas, con gran proporción de vehículos por habitante, y con más acciones de gestión ambiental (Cuadro 2). 
Cuadro 2 - Ciudades ricas del ACP.

\begin{tabular}{|l|c|c|}
\hline Ciudades ricas & Bioma & Región \\
\hline Araçatuba & Mata Atlántica & Sudeste \\
\hline Bento Gonçalves & Mata Atlántica & Sur \\
\hline Blumenau & Mata Atlántica & Sur \\
\hline Catanduva & Mata Atlántica & Sudeste \\
\hline Caxias do Sul & Mata Atlántica & Sur \\
\hline Florianópolis & Mata Atlántica & Sur \\
\hline Jundiaí & Mata Atlántica & Sudeste \\
\hline Maringá & Mata Atlántica & Sur \\
\hline Niterói & Mata Atlántica & Sudeste \\
\hline Poços de Caldas & Mata Atlántica & Sudeste \\
\hline Presidente Prudente & Mata Atlántica & Sudeste \\
\hline Santos & Mata Atlántica & Sudeste \\
\hline São Vicente & Mata Atlántica & Sudeste \\
\hline Vitória & Mata Atlántica & Sudeste \\
\hline Santa Cruz do Sul & Mata Atlántica/Pampa & Sur \\
\hline Americana & Mata Atlántica/Cerrado & Sudeste \\
\hline Bauru & Mata Atlántica/Cerrado & Sudeste \\
\hline Botucatu & Mata Atlántica/Cerrado & Sudeste \\
\hline Rio Claro & Mata Atlántica/Cerrado & Sudeste \\
\hline São José do Rio Preto & Mata Atlántica/Cerrado & Sudeste \\
\hline
\end{tabular}

Fuente: Elaboración propia.

En oposición a las ciudades pobres del Norte y Nordeste de Brasil, en las ciudades ricas predominan poblaciones adultas, con bajas tasas de natalidad y, además, más escolarizadas y con sueldos mayores. Por lo tanto, con estándares de consumo más sofisticados que aquellos presentes en las ciudades pobres. Los coches y otros vehículos ilustran bien las diferencias de consumo entre las ciudades medias pobres y ricas de Brasil. Las ciudades del Sur y Sudeste de Brasil tienen una relación vehículos por habitante muy similar a la de las ciudades de los países del primer mundo. En la ciudad de Maringá, por ejemplo, esa relación es de un vehículo privado para cada 2,08 habitantes. Codó, una de las ciudades pobres de nuestro análisis, tiene una relación de un vehículo para cada 17,71 habitantes y Cametá tiene un vehículo para cada 55,1 habitantes. 


\section{LAMOTORIZACIÓN Y SUS IMPACTOS EN LAS CIUDADES MEDIAS BRASILEÑAS}

En Brasil, los vehículos de las ciudades con más de 60.000 habitantes consumen aproximadamente 10 millones de toneladas equivalentes de petróleo. Además, en esas ciudades, el transporte individual es responsable por $74 \%$ del consumo de energía y del $80 \%$ de las emisiones de contaminantes nocivos para la salud humana. Los vehículos motorizados emiten 24 millones de toneladas de contaminantes anualmente, siendo el coste estimado de la contaminación de cerca de 1.600 millones de euros y el transporte privado responsable del $86 \%$ de este valor (MINISTÉRIO DAS CIDADES, 2004).

La baja relación ciudadanos/vehículo que hemos encontrado en las ciudades medias más prósperas apuntadas por nuestro análisis de componentes principales supone un importante problema de salud pública, por la contaminación emitida por vehículos (y la plétora de otros impactos asociados a su uso). Diferentemente de las décadas anteriores hoy, en las ciudades brasileñas, las fuentes móviles (es decir, vehículos con motores de combustión interna) son las principales emisoras de contaminación atmosférica (SALDIVA et al, 1994; MARTINS et al, 2001; ANGEOLETTO, 2012).

Se pueden resumir las consecuencias de la contaminación producida por vehículos para los ecosistemas urbanos (la motorización impone también impactos regionales y globales, como señalan, por ejemplo, SILVAet al, 2003) en dos: enfermedades diversas y daños a la biodiversidad urbana. Por ejemplo, el contaminante NOx reacciona con compuestos orgánicos volátiles, resultando en la formación de ozona, que es toxico a las plantas, animales y seres humanos (BOTKIN; BEVERIDGE, 1997; MARTINS et al, 2002).

Factores como estos indican la complejidad de planificar los ecosistemas urbanos. Alcanzar ciertos estándares de neguentropía dentro de los límites urbanos no solamente es una cuestión tecnológica, como nos quieren hacer creer algunos ingenieros e intelectuales optimistas en la capacidad humana para el progreso tecnológico. Las ciudades medias más motorizadas apuntadas por el ACP tienen, efectivamente, un desafío de una tajante magnitud, que comprende intersecciones entre salud pública, protección medioambiental, planificación y gestión ambiental urbana. En realidad, en mayor o menor grado, es este un dilema de todas las ciudades medias de Brasil, por la rápida y creciente expansión de la flota nacional de vehículos privados. 


\section{LA GESTIÓN AMBIENTAL EN CIUDADES MEDIAS DE BRASIL - CUADRO INSTITUCIONAL}

De las ciudades ricas apuntadas en el análisis de componentes principales, el 50\% poseían secretaría de medio ambiente exclusiva, o sea, no vinculada a otras secretarías y, por tanto, dedicadas exclusivamente a las cuestiones medioambientales. En el grupo de las ciudades pobres del ACP, ese porcentaje decrece a un 15\%. Para el universo de las 283 ciudades analizadas, sorprendentemente, solo un $25 \%$ de los municipios poseían secretarías de medio ambiente exclusivas. Las ciudades ricas poseen, proporcionalmente, más funcionarios actuando en cuestiones ambientales. Cuatro de las 20 ciudades pobres seleccionadas en nuestro estudio no tienen siquiera un único funcionario destinado al medio ambiente. No obstante, los funcionarios disponibles son pocos para ambos grupos de ciudades investigadas (Cuadros 3 y 4). Para el universo de las ciudades investigadas, el número medio de funcionarios era de 13 por grupo de 100.000 habitantes, siendo de esos, dos con carrera universitaria.

Cuadro 3 - Número de funcionarios municipales destinados a medio ambiente (las 10 más)

\begin{tabular}{|c|c|}
\hline $\begin{array}{c}\text { Número total de } \\
\text { funcionarios /100.000 habitantes }\end{array}$ & $\begin{array}{c}\text { Funcionarios con carrera } \\
\text { universitaria/100.000 habitantes }\end{array}$ \\
\hline Bauru: 58,21 & 1,58 \\
\hline Vitória: 51 & 8,89 \\
\hline Catanduva: 43,52 & Cero \\
\hline Presidente Prudente: 41,75 & 0,53 \\
\hline Florianópolis: 30,11 & 1,75 \\
\hline Araçatuba: 20,11 & 1,77 \\
\hline Santos: 22,51 & 6,22 \\
\hline Caxias do Sul: 19,7 & 1,94 \\
\hline Jundiaí: 10,5 & 6,21 \\
\hline São José do Rio Preto & 4,22 \\
\hline
\end{tabular}

Fuente: IBGE: Perfil dos Municípios Brasileiros - Meio Ambiente y elaboración propia. 
Cuadro 4 - Número de funcionarios municipales destinados a medio ambiente (las 10 menos).

\begin{tabular}{|c|c|}
\hline $\begin{array}{c}\text { Número total de } \\
\text { funcionarios } / 100.000 \text { habitantes }\end{array}$ & $\begin{array}{c}\text { Funcionarios con carrera } \\
\text { universitaria/100.000 habitantes }\end{array}$ \\
\hline Macapá: 25,77 & 1,4 \\
\hline Arapiraca; 18,77 & 0,54 \\
\hline Cametá: 18,07 & Cero \\
\hline Santarém: 10,28 & 1,9 \\
\hline Timon 6,73 & Cero \\
\hline São José de Ribamar: 2,37 & Cero \\
\hline Parintins; cero & Cero \\
\hline Castanhal: cero & Cero \\
\hline Codó: cero & Cero \\
\hline Itapipoca: cero & Cero \\
\hline
\end{tabular}

Fuente: IBGE: Perfil dos Municípios Brasileiros - Meio Ambiente y elaboración propia.

\section{ACCIONES DE GESTIÓN AMBIENTAL (FISCALIZACIÓN)}

Las acciones de fiscalización (resumidas en el Cuadro 5), en general, ocurren en un porcentaje reducido de las ciudades pobres seleccionadas por el análisis de componentes principales, en comparación a las ciudades ricas y al universo de ciudades estudiadas. No obstante, tres categorías de fiscalización se destacan por la baja ocurrencia, cualquiera que sea el grupo de ciudades: la fiscalización sobre la contaminación atmosférica producida por vehículos, la fiscalización de gasolineras y la fiscalización sobre el uso de fertilizantes y pesticidas en cultivos agrícolas.

\section{ACCIONES DE GESTIÓN AMBIENTAL (PROTECCIÓN DE RECURSOS NATURALES)}

Respecto a las acciones municipales de protección de recursos naturales (Cuadro 6), son especialmente preocupantes los bajos porcentuales de ciudades que practicaron la monitorización de la calidad y descontaminación del agua de cuerpos hídricos. Acciones de protección a los suelos agrícolas virtualmente no existieron. Acciones de control de la deforestación fueron frecuentes solamente en las ciudades ricas del ACP. Finalmente, pocas fueron las ciudades que, en la época de la recogida de datos promovida por el IBGE, ya habían elaborado sus planes de zonificación ecológico-económica, hecho que indica un bajo esfuerzo de planificación hacia el desarrollo sostenible. 


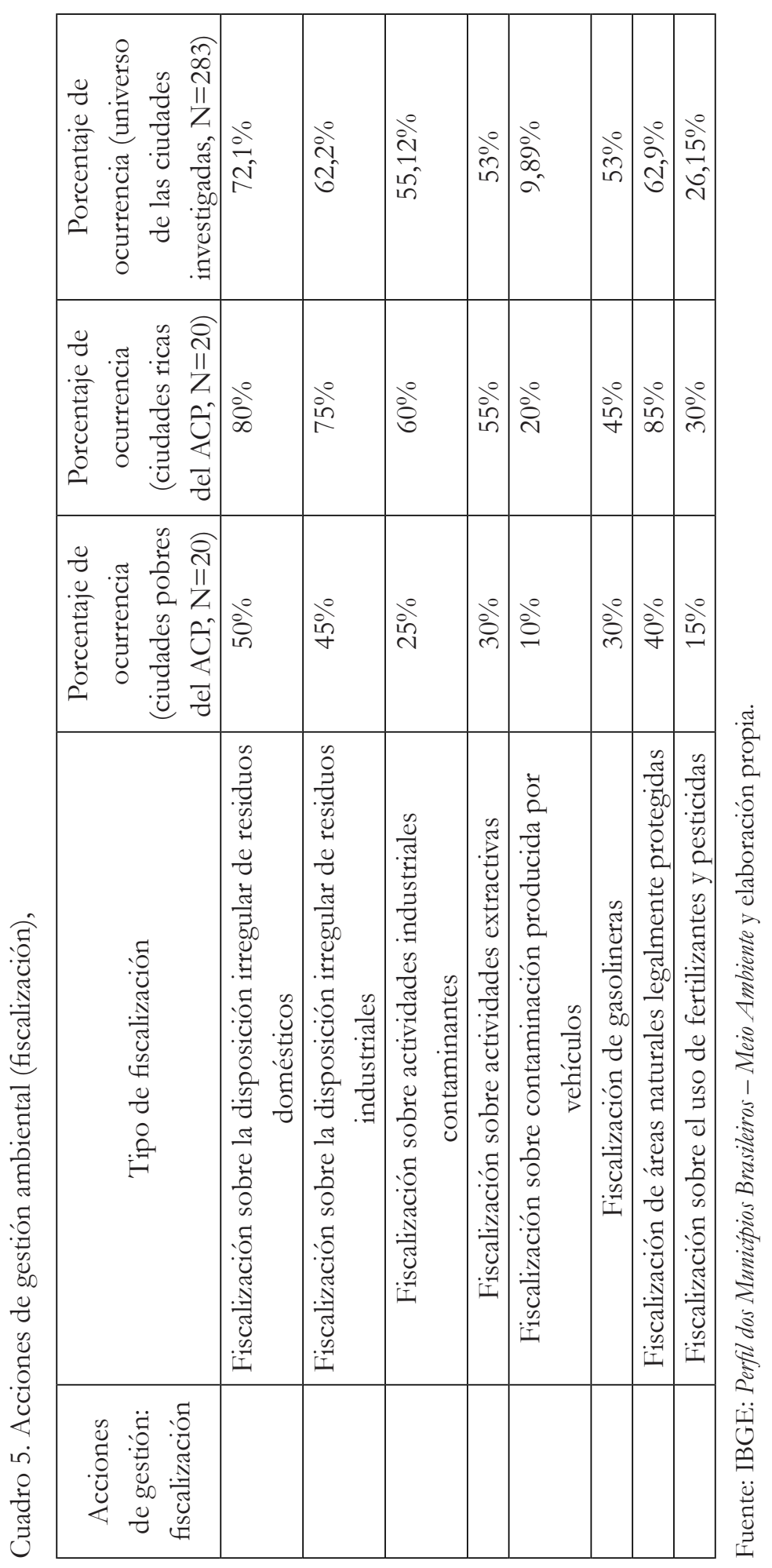




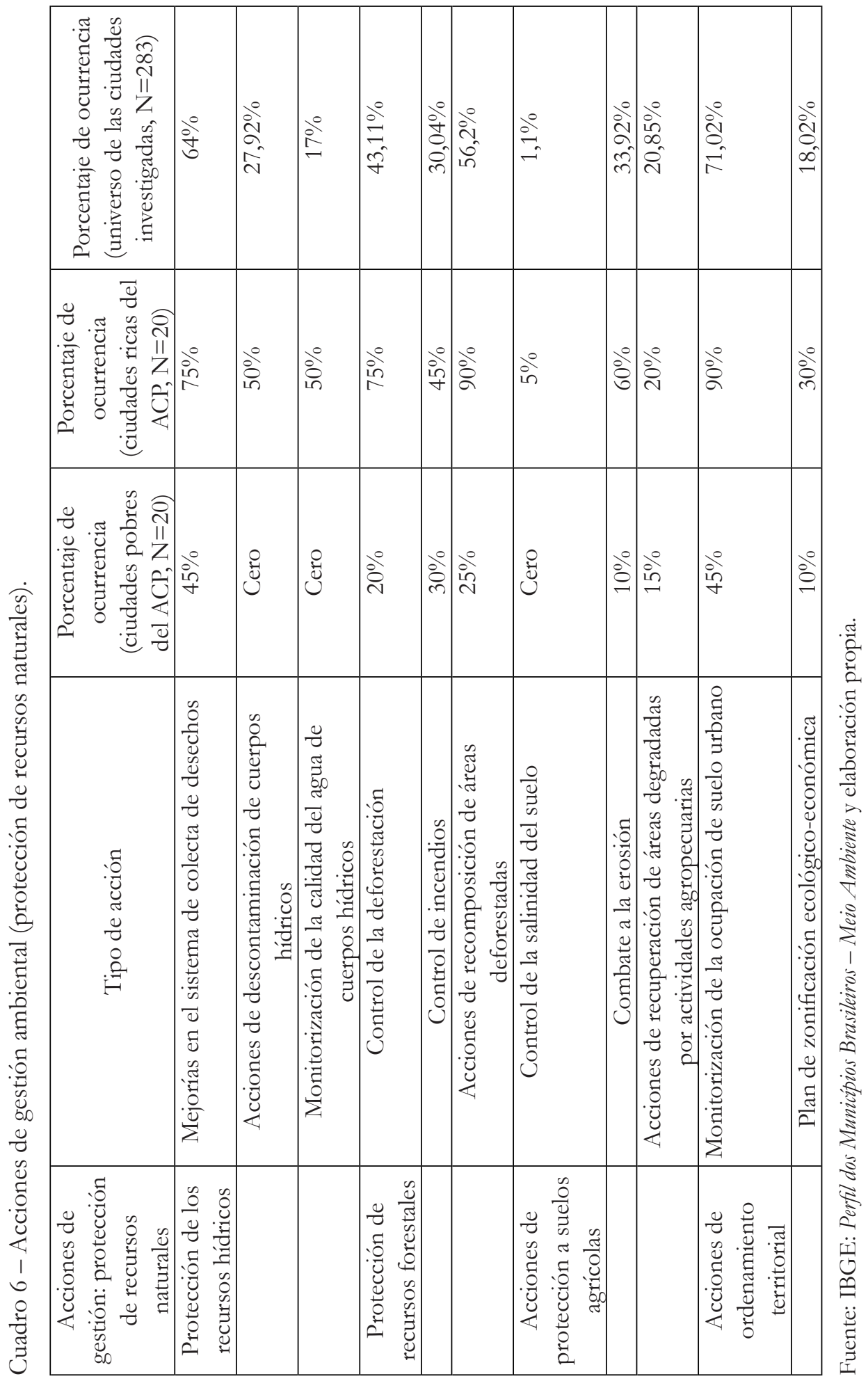




\section{PROGRAMAS DE MEDIO AMBIENTE MUNICIPALES}

La gran mayoría de las ciudades ricas del ACP (el 85\%) habían desarrollado programas de recogida selectiva de basura reciclable, aunque el IBGE no informe el porcentual de basura efectivamente captada por esos programas. Asimismo ha sido elevado el porcentaje de ciudades (el 68\%) donde se han desarrollado programas de educación ambiental, en el universo investigado. El desarrollo de programas agrícolas de control biológico de plagas y de producción de alimentos sin el uso de pesticidas y fertilizantes industriales (dos grandes fuentes de impactos ambientales) ha sido bajo, incluso en las ciudades ricas apuntadas a través del análisis de componentes principales (Cuadro 7).

Cuadro 7 - Acciones de gestión ambiental (programas ambientales)

\begin{tabular}{|c|c|c|c|c|}
\hline $\begin{array}{l}\text { Acciones } \\
\text { de gestión: } \\
\text { programas } \\
\text { ambientales }\end{array}$ & $\begin{array}{l}\text { Tipo de } \\
\text { programa }\end{array}$ & $\begin{array}{c}\text { Porcentaje de } \\
\text { ocurrencia } \\
\text { (ciudades } \\
\text { pobres del } \\
\text { ACP, } N=20 \text { ) }\end{array}$ & $\begin{array}{l}\text { Porcentaje de } \\
\text { ocurrencia } \\
\text { (ciudades } \\
\text { ricas del ACP, } \\
\quad \mathrm{N}=20 \text { ) }\end{array}$ & $\begin{array}{c}\text { Porcentaje de } \\
\text { ocurrencia } \\
\text { (universo de } \\
\text { las ciudades } \\
\text { investigadas, } \\
\mathrm{N}=283 \text { ) }\end{array}$ \\
\hline & $\begin{array}{c}\text { Recogida } \\
\text { selectiva de } \\
\text { basura reciclable }\end{array}$ & $10 \%$ & $85 \%$ & $54,1 \%$ \\
\hline & $\begin{array}{l}\text { Educación } \\
\text { ambiental }\end{array}$ & $40 \%$ & $75 \%$ & $67,84 \%$ \\
\hline & $\begin{array}{c}\text { Control } \\
\text { biológico de } \\
\text { plagas agrícolas }\end{array}$ & $10 \%$ & $25 \%$ & $21,91 \%$ \\
\hline & $\begin{array}{l}\text { Incentivo a } \\
\text { prácticas de } \\
\text { agricultura } \\
\text { orgánica }\end{array}$ & $25 \%$ & $40 \%$ & $35 \%$ \\
\hline
\end{tabular}

Fuente: IBGE: Perfil dos Municípios Brasileiros - Meio Ambiente y elaboración propia

\section{PRESENCIA DE EQUIPAMIENTOS PARA LA GESTIÓN AMBIENTAL URBANA}

En cuanto a los equipamientos para la gestión ambiental, la situación retratada por el IBGE, era, en el año de la recolección de datos, absolutamente preocupante. Con la excepción de la existencia de vertederos sanitarios 
(que faltaban a nada menos que un 42\% del conjunto de las 283 ciudades investigadas), los demás equipamientos han sido detectados en porcentuales aún más modestos. Lo mismo se verificó, también, respecto a la presencia de sitios para recibimiento de envases de pesticidas, aunque el consumo de este insumo agrícola fuera significativo en, respectivamente, $65 \%$ y $75 \%$ de las ciudades pobres $\mathrm{y}$ ricas seleccionadas por el análisis de componentes principales.

En relación al conjunto de ciudades investigadas, el consumo de pesticidas es significativo en $70 \%$ de los municipios. De modo semejante, pocas ciudades poseían vertederos para el recibimiento adecuado de residuos industriales, aunque la producción de residuos fuera significativa en $65 \%$ de las ciudades pobres del ACP, en $90 \%$ de las ciudades ricas y en $69 \%$ del total de ciudades analizadas. Solamente un $15 \%$ de los municipios pobres poseían unidades de conservación municipales (como parques y bosques), contra un $85 \%$ entre las ciudades ricas señaladas por el ACP. Por otro lado, la presencia de estaciones de medición de la calidad del aire no era común en los tres grupos de ciudades medias analizadas (Cuadro 8).

Cuadro 8 - Acciones de gestión ambiental (equipamientos para la gestión)

\begin{tabular}{|c|c|c|c|c|}
\hline $\begin{array}{c}\text { Acciones } \\
\text { de gestión } \\
\text { ambiental: } \\
\text { equipamientos }\end{array}$ & Equipamiento & $\begin{array}{c}\text { Porcentaje de } \\
\text { ocurrencia } \\
\text { (ciudades } \\
\text { pobres del } \\
\text { ACP, N=20) }\end{array}$ & $\begin{array}{c}\text { Porcentaje } \\
\text { de } \\
\text { ocurrencia } \\
\text { (ciudades } \\
\text { ricas del } \\
\text { ACP }, \mathrm{N}=20 \text { ) }\end{array}$ & $\begin{array}{c}\text { Porcentaje de } \\
\text { ocurrencia } \\
\text { (universo de } \\
\text { las ciudades } \\
\text { investigadas, } \\
\mathrm{N}=283 \text { ) }\end{array}$ \\
\hline & $\begin{array}{c}\text { Sitios para } \\
\text { recibimiento } \\
\text { de envases de } \\
\text { pesticidas }\end{array}$ & Cero & $35 \%$ & $21 \%$ \\
\hline & $\begin{array}{l}\text { Vertederos } \\
\text { industriales }\end{array}$ & Cero & $20 \%$ & $12 \%$ \\
\hline & $\begin{array}{l}\text { Vertederos } \\
\text { sanitarios }\end{array}$ & $55 \%$ & $65 \%$ & $58 \%$ \\
\hline & $\begin{array}{l}\text { Estaciones de } \\
\text { medición de la } \\
\text { calidad del aire }\end{array}$ & Cero & $15 \%$ & $11 \%$ \\
\hline & $\begin{array}{l}\text { Unidades de } \\
\text { conservación } \\
\text { municipales }\end{array}$ & $15 \%$ & $85 \%$ & $59 \%$ \\
\hline
\end{tabular}

Fuente: IBGE: Perfil dos Municípios Brasileiros - Meio Ambiente y elaboración propia 


\section{GESTIÓN AMBIENTAL DE LAS CIUDADES MEDIAS: NI SIQUIERA UNA SOSTENIBILIDAD BLANDA}

La escasez de funcionarios dedicados al medio ambiente, así como la relativa poquedad de equipamientos y acciones de gestión ambiental en las ciudades medias de Brasil, sugieren un cuadro de poca efectividad ante los problemas e impactos ambientales que necesitan ser corregidos o evitados en esas ciudades. Muchos de los problemas ambientales urbanos resultan de administraciones ineficaces, de la falta de planificación y políticas urbanas coherentes, más que del proceso de urbanización per se (HARDOY et al, 2001), y en las ciudades brasileñas la gestión y planificación todavía no han sido herramientas ambientalmente efectivas (GRIMM et al 2008; ANGEOLETTO, 2012). Algunos economistas y académicos argumentan que el crecimiento económico acaba por generar un considerable volumen de recursos para gastos en políticas de protección ambiental (LEVESON-GOWER, 1997), critica esta argumentación de modo contundente), pero los resultados de nuestra investigación señalan un cuadro distinto, ya que las acciones de gestión ambiental en las ciudades medias que hemos investigado, de manera general, son débiles y de eficacia discutible. Esto ocurre no solo en las ciudades pobres, con fuertes demandas sociales que disminuyen aun más los pocos recursos disponibles, sino también en las ciudades medias ricas del Sur y Sudeste de Brasil, donde esas acciones son más frecuentes. Efectivamente, según Dodds (1997), la relación entre el aumento de la renta y una mayor demanda por calidad ambiental ha sido establecida solamente para un conjunto limitado de cuestiones ambientales (por ejemplo bajos niveles de contaminantes en el aire y el agua), en ciudades de naciones ricas. Es lo que el ecólogo José Luis Rubio de Lucas, de la Universidad Autónoma de Madrid, define como sostenibilidad blanda (José Luis Rubio de Lucas, comunicación personal, 2014).

Todas las ciudades medias están ubicadas en biomas de megabiodiversidad. De estas, destacamos aquellas (totalizando 28 ciudades) ubicadas en los biomas Mata Atlántica y Cerrado, que son hotspots de biodiversidad, es decir, biomas de elevada diversidad biológica, que contienen un largo número de especies endémicas y que se encuentran bajo extrema amenaza [por factores como urbanización, agricultura y otros] (MITTERMEIER et al, 1998). Además, siete de las ciudades del ACP están ubicadas en zonas de transición entre biomas, los ecotonos, por lo que, desde el punto de vista de la conservación de la diversidad biológica, son especialmente relevantes. Son ellas Arapiraca (en la transición entre los biomas Mata Atlántica/Caatinga); Timon (Cerrado/Caatinga) Americana, Bauru, Botocatu, Rio Claro y São José do Rio Preto (Mata Atlántica/Cerrado); y Santa Cruz do Sul (Mata Atlántica/Pampa). 
Smith y colaboradores (1997) al analizar poblaciones de especies comunes de pájaros de un bosque pluvial y de un ecotono (bosquepluvial/ sabana), detectaron diferencias morfológicas entre las poblaciones del bosque y del ecotono, a pesar del intenso flujo génico entre ellas. Esos datos evidencian la posibilidad de que los ecotonos sean sitios de especiación, por lo que se recomienda una mayor atención a la conservación de esas zonas de transición (SMITH et al, 2001). Así que, para las ciudades medias ubicadas en ecotonos, habría que destinarse recursos para proyectos de investigación aplicables a la planificación y gestión ambiental urbanas que redundasen en menos impactos a la diversidad biológica de esos hábitats.

Respecto a las acciones de fiscalización, es especialmente preocupante el bajo porcentaje de ciudades que practican la fiscalización sobre la contaminación atmosférica producida por vehículos, sobre las gasolineras y sobre el uso de fertilizantes químicos y pesticidas. Hay en Brasil más de 35.000 gasolineras. De esas, 8.000 están ubicadas en el Estado de São Paulo (LOPES et al, 2010), donde se ubica el 29\% de las ciudades medias de Brasil. La gasolina contiene varios contaminantes, entre ellos, benceno, tolueno, etilbenceno y xilenos (conocidos pela sigla BTEX). Los BTEX son extremamente tóxicos. Causan depresión del sistema nervioso central y son carcinogénicos. La gasolina brasileña posee una peculiaridad: se trata efectivamente de una mezcla de $80 \%$ de gasolina y $20 \%$ de etanol (por fuerza de ley federal). Así que, los BTEX son más solubles en etanol, y a su vez, el etanol es muy soluble en agua, el que aumenta la contaminación, en caso de vaciamientos (CORSEUIL; MARINS, 1997; SILVA et al, 2002).

País que figura entre los más importantes players de la agroindustria global, Brasil es uno de los mayores consumidores de pesticidas del mundo (FARIA et al, 2007). Entre 1960 y 2000, el área cultivada en Brasil ha aumentado un $78 \%$, mientras que el consumo de pesticidas ha crecido en 700\% (SPADOTTO, 2006). Las ventas de pesticidas se concentran principalmente en los estados de Rio Grande do Sul, Paraná y São Paulo (ALVES FILHO, 2002), donde están ubicadas el 44\% de las ciudades medias brasileñas y 15 de las 20 ciudades ricas señaladas por el ACP. Los bajos porcentuales de ciudades con sitios apropiados para el recibimiento de envases usados de pesticidas (ninguna de las ciudades pobres señaladas por el ACP los poseen, aunque el consumo de pesticidas sea significativo en 65\% de ellas) y la poca fiscalización del uso de esos venenos, asimismo como en el caso de las gasolineras, presupone impactos al ambiente y a la salud pública. Por desgracia, los órganos públicos responsables por la fiscalización del uso de pesticidas son débiles y no presentan condiciones siquiera de reunir y evaluar datos sobre su consumo (ALVES FILHO, 2002). 


\section{CONSIDERACIONES FINALES}

En un momento de descentralización política de ámbito mundial, crecen los servicios y responsabilidades (incluso de orden ambiental) que asumen las ciudades, pero raramente las municipalidades tienen personal preparado y capacidad técnica y administrativa para ellos. Por ello, hay que echar mano de ideas innovadoras y poco costosas e implantarlas, a través del capital humano disponible. No nos referimos solamente al personal técnico de los ayuntamientos de las ciudades medias, sino también a la participación popular en la planificación y desarrollo de los proyectos. Además, la poquedad de funcionarios en las secretarías municipales de medio ambiente de los ayuntamientos puede ser resuelta, por lo menos en parte, con la inclusión de técnicos de otras instituciones, como las universidades. La planificación y gestión adecuadas de las ciudades son tan importantes a la conservación de la biodiversidad cuanto es el establecimiento de áreas naturales legalmente protegidas (BOTKIN; BEVERIDGE, 1997; PNUMA, 2011). De hecho, aunque el porcentual de áreas protegidas esté aumentando mundialmente desde 1990, el número de especies amenazadas sigue creciendo (PNUMA, 2011), hechos que ponen de relieve la importancia de la planificación y gestión urbanas orientadas a la disminución de impactos ambientales causados por las ciudades.

Como cantó el bardo Bob Dylan, beauty walks a razor's edge. Queda claro que, si bien las cuestiones ambientales han alcanzado importancia en la sociedad civil, poca prioridad es dada a los problemas ambientales en ámbito municipal, en Brasil. Ni siquiera hemos alcanzado, en las ciudades medias brasileñas, los estándares de sostenibilidad blanda de ciudades de países desarrollados (es decir, sostenibilidad para unos pocos parámetros, como los relativos a la calidad del agua y aire en ambientes urbanos). ¿Cuándo habrá de hecho una agenda para las cuestiones medioambientales en el ámbito municipal en Brasil? Hay mucho que hacer, para que no repitamos en las ciudades medias las innumerables tragedias ambientales omnipresentes en las metrópolis brasileñas.

AGRADECIMIENTOS: Al Ministerio de Agricultura, Alimentación y Medio Ambiente del gobierno español, por la financiación al Proyecto Ecología Urbana en Regiones Metropolitanas de Brasil: Paisaje, Calidad de Vida y Desarrollo Humano [Plan Nacional de I+D+i (CSO2009-12689)].

\section{REFERENCIAS}

ALVES FILHO, J.P. Uso de Agrotóxicos no Brasil - Controle Social e Interesses

Corporativos. São Paulo: Annablume, 2002. 
ANGEOLETTO, F. Planeta Ciudad: Ecología Urbana y Planificación de Ciudades Medias de Brasil. 280p.Tesis doctoral, Departamento de Ecología, Universidad Autónoma de Madrid, Madrid, 2012.

BOTKIN, D.B.; BEVERIDGE, C.E. Cities as environments. Urban Ecosystems,n.1, p.3-19, 1997.

CARVALHO, E. Exclusão social e crescimento das cidades médias brasileiras. Scripta Nova - Revista Electrónica de Geografía y Ciencias Sociales, v.7, n.146, 2003.

COHEN, J. Human Population: The Next Half Century. Science, v.302 p.1172$1175,2003$.

CORSEUIL, H.X.; MARINS, M.D.M. Contaminação de águas subterrâneas por derramamento de gasolina: o problema é grave? Revista Engenharia Sanitária e Ambiental, v.2, n.2, p.50-54, 1997.

DODDS, S. Economic Growth and Human Well-being. In Diesendorf, M.; Hamilton, C.; (eds.). Human Ecology, Human Economy. Sidney: Allen \& Unwin, 1997.

FARIA, N.; FASSA, A.C.; FACCHINI, L. Intoxicação por agrotóxicos no Brasil: os sistemas oficiais de informação e desafios para realização de estudos epidemiológicos. Ciência \& Saúde Coletiva, v.12, n.1, p.25-38, 2007.

HARDOY, J.E.; MITLIN, D.; SATTERTHWAITE, D. Environmental Problems in a Urbanizing World: Finding Solutions in Africa, Asia and Latin America. Londres: Earthscan, 2001.

IBGE. Perfil dos Municípios Brasileiros - Meio Ambiente, 2002. Rio de Janeiro: IBGE, 2005.

IPEA. Comunicados do IPEA, no 68 - Análise Preliminar dos dados do Censo 2010. Brasília: IPEA, 2011.

GRIMM, N.B.; FAETH, S.H.; GOLUBIEWSKI, N.E. et al. Global change and the ecology of cities. Science, v.319, n.5864, p.756-760, 2008.

LEVESON-GOWER, H. Trade and the Environment. In: Diesendorf, M.; Hamilton, C. (eds.). Human Ecology, Human Economy.Sydney: Allen \& Unwin Publishers, 1997.

LOPES, M.B.; SILVA, A.L.; CONEJERO, M.A. Fluxos e poder nos canais de distribuição de etanol carburante: um estudo qualitativo no estado de São Paulo. Revista de Administração, v.45, n.4, p.356-372, 2010.

MARTINS, L.C.; LATORRE, M.R.D.O.; SALDIVA, P.H.N.; (et al). Relação entre poluição atmosférica e atendimentos por infecção de vias aéreas superiores no município de São Paulo: avaliação do rodízio de veículos. Revista Brasileira de Epidemiologia, v.4, n.3, p.220-229, 2001.

MARTINS, L.C.; LATORRE, M.R.D.O; CARDOSO, M.R.A. (et al). Poluição 
atmosférica e atendimentos por pneumonia e gripe em São Paulo, Brasil. Revista de Saúde Pública, v.36, n.1, p.88-94, 2002.

MINISTÉRIO DAS CIDADES. Perfil da mobilidade, do transporte e do trânsito nos municípios brasileiros. Brasília: Ministério das Cidades, 2004.

MITTERMEIER, R.A.; MYERS, N.; THOMSEN, J.B. et al. Biodiversity hotspots and major tropical wilderness areas: approaches to setting conservation priorities. Conservation Biology, v.12, n.3, p.516-520, 1998.

PNUMA Programa de las Naciones Unidas para el Medio Ambiente. Anuário PNUMA - Temas Emergentes en Nuestro Medio Ambiente Global. Nairobi: PNUMA, 2011.

PRADO, P.I.; LEWINSOHN, T.M.; CARMO, R.L. et al. Ordenação Multivariada na Ecologia e seu Uso em Ciências Ambientais. Ambiente e Sociedade, v.5, n.10, p.69-83, 2001.

REES, W. E. Urban Ecosystems: The Human Dimension. Urban Ecosystems, n.1, p.63-75, 1997.

SALDIVA, P.H.N.; LICHTENFELS, P.S.O.; PAIVA, I.A. et al. Association between air pollution and mortality due to respiratory diseases in children in São Paulo, Brazil: a preliminary report. Environmental Research, n.65, p.218-225, 1994.

SILVA, R.L.B.; BARRA, C.M.; MONTEIRO, T.C.N. et al. Estudo da contaminação de poços rasos por combustíveis orgânicos, e possíveis conseqüências para a saúde pública ao município de Itaguaí, Rio de Janeiro, Brasil. Cadernos de Saúde Pública, v.18, n.6, p.1599-1607, 2002.

SMITH, T.B.; WAYNE, R.K.; GIRMAN, D.J.; et al. A role for ecotones in generating rainforest biodiversity. Science, v.276, n.5320, p.1855-1857, 1997.

SMITH, T. B., KARK, S., SCHNEIDER, C. J., WAYNE, R. K., AND MORITZ, C. Biodiversity hotspots and beyond: the need for preserving environmental transitions. Trends in Ecology and Evolution, v.16, n.8, p.431, 2001.

SILVA, J.M.C.; TABARELLI, M.; FONSECA, M.T. et al. Biodiversidade da caatinga: Áreas e Ações Prioritárias para a Conservação. Brasília: MMA, 2003.

SPADOTTO, C.A. Avaliação de riscos ambientais de agrotóxicos em condições brasileiras. Embrapa Meio Ambiente: Documentos, n.58, p.2-22, 2006.

TERRADAS, J. Ecología Urbana. Barcelona: Editorial Rubes, 2001.

UNITED NATIONS HUMAN SETTLEMENTS PROGRAMME (UN-HABITAT). State of the World's Cities 2008/2009. Londres: Earthscan, 2008. WORLDWATCH INSTITUTE. Estado do Mundo 2005 - Estado do Consumo e Consumo Sustentável. Salvador: UMA Editora, 2006. 